\title{
Experimental Investigation of Resistive Sensor for High Power Millimetre Wave Pulse Measurement
}

\author{
R. SimnišKis*, M. DAgYs, Ž. KANCleRis And V. TAMošIŪNAS \\ Semiconductor Physics Institute
}

A. Goštauto 11, LT-01108, Vilnius, Lithuania

\begin{abstract}
An experimental investigation of a high power millimetre wave pulse sensor is presented. Two groups of sensors with a flat frequency response were fabricated according to results of the optimisation. The frequency response and voltage standing wave ratio were measured and compared with results of the electrodynamical simulations. The largest measured sensitivity variation of the best sensors within waveguide frequency range was $\pm 8 \%$. The reasonable agreement between measured and simulated values of the sensitivity was obtained.
\end{abstract}

PACS numbers: 81.05.-t, 81.05.Rm, 81.05.Ea

\section{Introduction}

High power millimetre wave (HPMW) sources very often operate in a pulsed mode. Modern trends of pulsed HPMW applications are to generate shorter pulses and higher power [1], meanwhile there are no commercial high power pulsed millimetre wave sensors. Therefore, the development of HPMW pulse sensors is a problem of great importance.

A resistive sensor (RS), the performance of which is based on electron heating effect, was successfully applied in the microwave bands from $1 \mathrm{GHz}$ up to $40 \mathrm{GHz}$. The RS has demonstrated its advantage over the diode at high power levels [2]. Unfortunately, the downscaling of the well-designed centimetre wave RS with diaphragm to millimetre wave range was not possible due to small dimensions. Therefore, a new concept of the RS has been proposed for HPMW pulse measurement $[3,4]$. A three-dimensional finite difference time domain (FDTD) calculations have been performed to determine the dimensions and specific resistance of the sensor providing nearly constant sensitivity in the waveguide's frequency range. In this contribution, practical realisation of ideas proposed in $[3,4]$ is presented.

${ }^{*}$ corresponding author; e-mail: rimsim@pfi.lt 


\section{Concept of resistive sensor}

The structure proposed for HPMW pulse application is shown in Fig. 1. It consists of two sensing elements made from $n$-Si mounted in the middle of the wide wall of the waveguide, upper contacts of which are shortened with a metal foil. Basing on the results of simulations [3] two groups of sensors were manufactured from different specific resistance $\rho$ of $n$-Si: the first group was made from $\rho=1 \Omega \mathrm{cm}$, the second - from $2 \Omega \mathrm{cm}$. The dimensions of both types

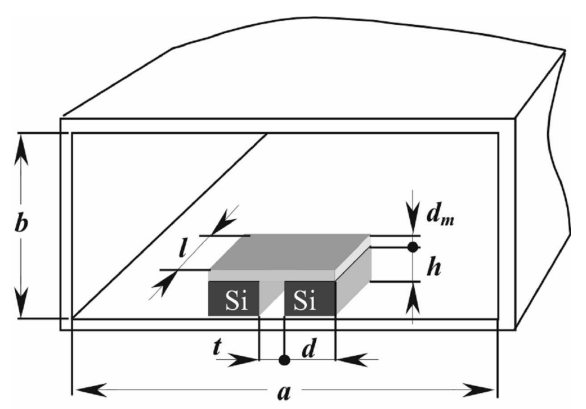

Fig. 1. Sketch of the RS for high power millimetre wave pulse measurement in the waveguide.

sensors were $h=0.1 \mathrm{~mm}, d=0.15 \mathrm{~mm}, l=0.6 \mathrm{~mm}$. Sensors were mounted in the $W$ band waveguide $(78-118 \mathrm{GHz}) a=2.4 \mathrm{~mm}, b=1.2 \mathrm{~mm}$. The resistance of the first group sensors was roughly $20 \Omega$, whereas of the second $-45 \Omega$. The regular thickness of shortening metal foil $d_{\mathrm{m}}$ was $50-75 \mu \mathrm{m}$. The air gap between sensors $t$ was of the order of $100 \mu \mathrm{m}$.

\section{High power measurements}

The dependences of the output signal on the pulse power were measured up to $2 \mathrm{~kW}$ using a magnetron operating at $94 \mathrm{GHz}$. The duration of millimetre wave pulses was $300 \mathrm{~ns}$. In order to get high output signal, the RS was fed by a pulsed current source producing $10 \mathrm{~V}$ voltage drop on the sensing elements. At a maximum pulse power, the output signal was roughly $1.5 \mathrm{~V}$ for the RS of the first group and exceeded $2 \mathrm{~V}$ for the second group. From the experimental data the dependence of the relative resistance change of the $\mathrm{RS}$ on the pulse power was determined.

These results are shown in Fig. 2. It is seen that at a maximum pulse power the largest value of $\Delta R / R$ was about $20 \%$ for the first group of the RS and $50 \%$ for the second. As one might expect in a high power region the dependence of $\Delta R / R$ on $P$ deviates from the linear dependence predicted by (1). It is well established that dependence of $\Delta R / R$ on the pulse power in a wide range of $P$ can be approximated by the second order polynomial [2] with two parameters that are determined from the fitting procedure. Two term approximations are shown 
in Fig. 2 by solid lines. It is seen that the considered approximation fits well the experimental data. From the linear part of the dependence of $\Delta R / R$ on $P$, the values of the coefficients $\zeta$ were determined.

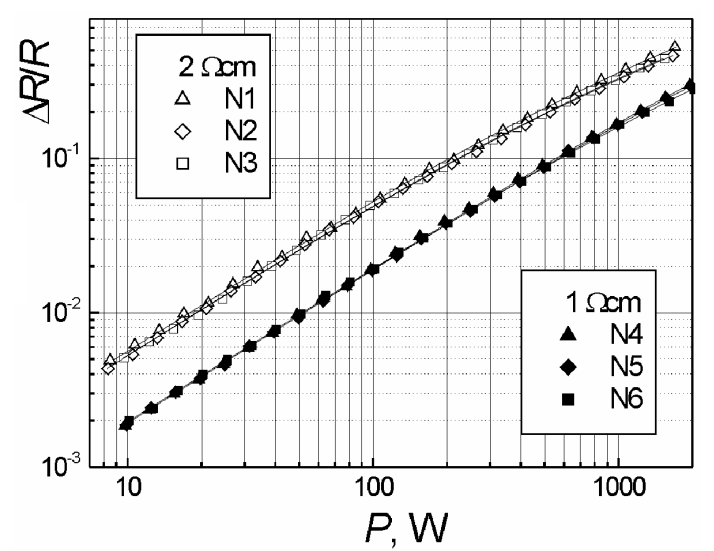

Fig. 2. The dependence of the relative resistance change on the pulse power.
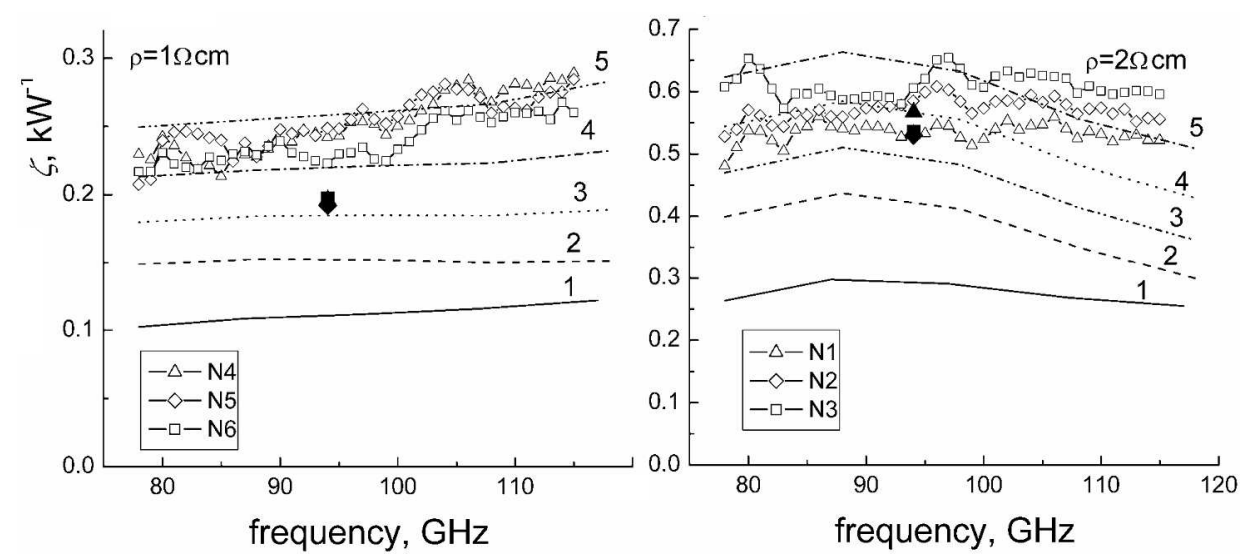

Fig. 3. Dependences of the sensitivity on frequency. Open points correspond to the measurement with the low power tunable generator, solid points - measurement with the magnetron generator. Lines - simulation for different $t$ and $d_{\mathrm{m}}: 1-t=d_{\mathrm{m}}=0$; $2-t=0.1 \mathrm{~mm}, d_{\mathrm{m}}=0 ; 3-t=0.1 \mathrm{~mm}, d_{\mathrm{m}}=25 \mu \mathrm{m} ; 4-t=0.1 \mathrm{~mm}, d_{\mathrm{m}}=50 \mu \mathrm{m}$; $5-t=0.1 \mathrm{~mm}, d_{\mathrm{m}}=75 \mu \mathrm{m}$.

They are shown in Fig. 3 by solid points. The better coincidence between the results measured using different techniques was obtained for the second group of the RS. It might be attributed to the fact that the second group of sensors are more sensitive and consequently the better measurement accuracy should be characteristic of this group of RS. 


\section{Sensitivity and voltage standing wave ratio measurements}

One of the main characteristics of the RS is its sensitivity $\zeta$, defined as a ratio of a relative resistance change $\Delta R / R$ to power $P$ transmitted through the waveguide

$$
\zeta=\frac{\Delta R / R}{P}
$$

It is worth to mention that $\zeta$ describes sensitivity in a low power limit, where the relative resistance change linearly depends on power [2].

The sensitivity, voltage standing wave ratio (VSWR), and insertion loss dependences on frequency have been measured using low power tunable millimetre wave source, reference wattmeter and lock-in amplifier. It was found that VSWR is less than 1.25 and insertion loss - $0.8 \mathrm{~dB}$ for the RS of both groups in a frequency range $78-118 \mathrm{GHz}$. Results of the measurements of the dependence of the sensitivity on frequency with results of the simulations are presented in Fig. 3. It is seen that the measured sensitivity variation was $\pm 15 \%$ for the first group and $\pm 8 \%$ for the second. Taking into account the finite thickness of the metal foil $d_{\mathrm{m}}$ and an air gap $t$ between the sensing elements (Fig. 1) in the simulations, the reasonable agreement between measured and calculated values of the sensitivity was obtained.

Concluding, we would like to emphasise that the reproducible RS for the millimetre wave range have been developed. They demonstrate flat frequency response, have low VSWR, and produce high output signal. The performed investigations have confirmed that the developed RS can be efficiently used for the measurement of the HPMW pulses.

\section{Acknowledgments}

Lithuanian State Science and Studies Foundation supported the work.

\section{References}

[1] M. Thumm, Int. J. Infrared Millim. Waves 26, 483 (2005).

[2] M. Dagys, Ž. Kancleris, R. Simniškis, E. Schamiloglu, F. Agee, IEEE Antennas Propag. Mag. 43, 64 (2001).

[3] Ž. Kancleris, V. Tamošiūnas, M. Dagys, R. Simniškis, F.J. Agee, IEE Proc.Microwaves Anten. Propag. 152, 240 (2005).

[4] V. Tamošiūnas, Ž. Kancleris, M. Dagys, R. Simniškis, F.J. Agee, Acta Phys. Pol. A 107, 420 (2005). 